\title{
An Anomalous dust emission component? - the observations
}

\section{R.D. Davies*}

University of Manchester, Jodrell Bank Observatory

Macclesfield, Cheshire SK119DL, UK

E-mail: rdd@jb.man.ac.uk

\begin{abstract}
This contribution describes the observations of the anomalous emission from interstellar dust at radio and millimetre wavelengths. Complementary information is available from data sets ranging from all-sky surveys to high resolution studies of individual clouds. The large area surveys use cross-correlation or MEM techniques to infer some properties of the emission; it is the major foreground to the $\mathrm{CMB}$ in the frequency range 20-60 GHz, exceeding the free-free and synchrotron contributions.

The physical processes involved are best confronted by studies of individual clouds. For example the anomalous dust emissivity varies from cloud to cloud at $T_{D}^{1.6}$ and the tightest correlation is with the 25 micron emission. The spectrum of the clouds is similar to that expected for spinning dust. The first indications are that the anomalous emission is unpolarized $(<5 \%)$. These properties strongly indicate that very small grains are responsible for this emission.
\end{abstract}

CMB and Physics of the Early Universe - International Conference

Ischia, Italy

April $20-22,2006$

\section{${ }^{*}$ Speaker}




\section{Introduction}

Dust is becoming increasingly recognised as an important foreground to the cosmic microwave background (CMB). In the last 10 years a new emission component of dust has been identified which dominates over the other foregrounds (free-free and synchrotron) at frequencies from $\sim 10$ to $\sim 60 \mathrm{GHz}$; the thermal (vibrational) dust dominates at the higher frequencies. It is of high interest to understand the properties of this new dust emission mechanism in order to construct a process (a template or whatever) both to remove this significant foreground to the CMB over most of the sky and also to unravel the physics of the emission process itself.

The first detections of a dust-correlated emission were misinterpreted as being free-free emission on the reasonable assumption that gas, both neutral and ionized was generally thought to be roughly coupled to dust in the general interstellar medium (ISM). The COBE-DMR maps (Kogut et al. 1996a,b) showed emission correlated with dust (240 $\mu$ emission) which had a freefree spectrum between 31 and $53 \mathrm{GHz}$ and were interpreted as such. de Oliveira-Costa et al. (1997) interpreted the $40 \mathrm{GHz}$ Saskatoon data in a similar way. An experiment well-placed in frequency (at 14.5 and $31.7 \mathrm{GHz}$ ) to detect this was the deep scan around the NCP by Leitch et al. $(1997,2000)$ which showed a strong correlation with $100 \mu$ dust and a spectrum not inconsistent with free-free (and synchrotron). Since no strong $\mathrm{H} \alpha$ emission was evident in the area, this was interpreted as free-free from a $\mathrm{T}_{\mathrm{e}} \sim 10^{6} \mathrm{~K}$ gas which suppressed the $\mathrm{H} \alpha$ emission. The above data were just what Draine \& Lazarian (1998) needed to verify their model of excess emission at several $10 \mathrm{GHz}$ from electric dipole rotational emission of very small grains.

The spinning dust paradigm has been increasingly accepted as the explanation of the anomalous emission. The situation nevertheless is not entirely clear. Other mechanisms operating within dust grains may also contribute. Draine \& Lazarian (1999) also considered an enhancement of thermal emissivity produced by thermal fluctuations in the grain magnetization. Further proposals for dust grain emission at low temperatures involve tunnelling in two-level systems(Agladze et al. 1996, 1998 and see L. Verstraete in this volume).

This paper will review the observations of anomalous emission from dust in various environments, ranging from diffuse emission at intermediate and high Galactic latitudes, to isolated structures and compact dust clouds as well as dust in HII regions. These data are already providing critical clues as to the processes and the environments conducive to generating the anomalous emission.

\section{Anomolous emission from the widespread diffuse dust}

The first demonstration that the dust itself could be the origin of this extra emission was by Draine \& Lazarian (1998) who showed that small spinning dust grains would emit electric dipole radiation in the frequency range $10-60 \mathrm{GHz}$ and could explain the dust-correlated emission reported by Kogut et al. (1996), de Oliveira-Costa et al. (1997) and Leitch et al. (1997). The emitting grains would be small, containing $\sim 100$ atoms, and would be 
characterized by emission in the IRAS $10-25 \mu$ bands. The part of the spinning dust spectrum immediately above the peak of the spectrum at $\sim 30 \mathrm{GHz}$ has a power spectral index in the range -0.2 to -0.6 which makes it indistinguishable from free-free and synchrotron emission. The falling spectrum below the peak at $20 \mathrm{GHz}$ provides the distinguishing characteristic of spinning dust; for the diffuse ISM this cannot be confused with free-free emission although one needs to be aware of optically thick HII regions.

The first indication of the spinning dust low-frequency turn-over came from combining the Tenerife data with COBE-DMR data (de Oliveira-Costa et al. 1999). This showed a peak in the power spectrum at $\sim 20 \mathrm{GHz}$ consistent with spinning dust for the $5000 \mathrm{sq}$ degrees of the Tenerife intermediate latitude survey. This result was more clearly confirmed when comparing the Tenerife data with the WMAP data (de Oliveira-Costa et al. 2004); the low frequency results were inconsistent with a synchrotron spectrum for this diffuse intermediate latitude dust.

Banday et al. (2003) made the first all-sky analysis of the COBE-DMR data set based on a simultaneous cross-correlation with the free-free, synchrotron and dust all sky maps. The freefree template was the H $\alpha$-derived map of Dickinson, Davies \& Davis (2003), the synchrotron template was the $408 \mathrm{MHz}$ map of Haslam et al. (1982) while the dust template was the DIRBE 140 micron map. All data were converted to the COBE-DMR resolution of $7^{\circ}$. The COBEDMR frequencies of 31.5, 53 and $90 \mathrm{GHz}$ were supplemented by the Boughn et al. (1992) map at $19.2 \mathrm{GHz}$ to help define the synchrotron and dust-correlation emission spectral indices. Over the frequency range 19.2 to $90 \mathrm{GHz}$ the dust-correlated emission had a (temperature) spectral index of $\beta=-2.5$ which was synchrotron-like; inclusion of Tenerife data showed however a flattening at 10 and $15 \mathrm{GHz}$. The application of a reliable free-free template improved the anomalous dust fit by a factor of 3 compared with Kogut et al. (1996). More extensive low frequency data was still required to make a definitive confirmation of the spinning dust origin of anomalous emission. Nevertheless this anomalous emission was found to be the dominant emission in the $31.5 \mathrm{GHz}$ COBE-DMR band.

The WMAP team (Bennett et al. 2003) have come to radically different conclusions about the dust-correlated emission which they assert to be synchrotron emission with a thermal dust morphology. Their analysis showed that less than $5 \%$ of the $33 \mathrm{GHz}$ emission was from spinning dust with a spectrum of the form proposed by Draine \& Lazarian (1998). This view was reaffirmed in the MEM analysis of the 3-year data (Hinshaw et al. 2006) on a $1^{\circ}$ scale by their assertion that the synchrotron emission was remarkably well correlated with thermal dust emission in active starforming regions. They derived a flat synchrotron spectral index of about -2.4; by contrast the normal steep-spectrum $408 \mathrm{MHz}$ correlated synchrotron had a spectral index of -2.73 between $408 \mathrm{MHz}$ and $23 \mathrm{GHz}$. The 3 year WMAP team (Page et al. 2006) find that their two synchrotron components have different levels of linear polarization - the normal synchrotron has high polarization and the dust-correlated has low (undetected) polarization. This would seem to put a strain on a synchrotron origin for the latter component. 
A different perspective on the anomalous dust emission detected by WMAP has been provided by Lagache (2003) who looked at the WMAP millimetre emissivity as a function of HI surface density. The high latitude $\left(|b|>15^{\circ}\right)$ relative emissivity was a factor of $\sim 5$ less in the higher HI density regions, following a similar trend to that found for small transiently heated grains. This work suggests that the anomalous emission is associated with the very small transiently heated dust particles rather than the large grains responsible for the thermal dust emission.

\section{Isolated dust features}

I now consider the observations of isolated dust features rather than the average properties of large areas of sky. One of the first demonstrations of excess radio emission clearly associated with 100 micron dust and HI was the data of Leitch et al. (1997, 2000). Using a beamwdith of 7 arcmin at 14.5 and $32 \mathrm{GHz}$ they found a close correlation with $100 \mu$ dust features in the NCP dust and HI Loop. Although it was not recognized in the first publication as anomalous dust emission, it is an important data set which shows anomalous emission in a region of low star formation activity and low $325 \mathrm{MHz}$ synchrotron emission on the relevant scales.

Davies et al. (2006) used the WMAP data base to study the $\mathrm{cm} / \mathrm{mm}$ spectrum of freefree, synchrotron and dust emission. 15 regions at intermediate Galactic latitude were selected in each of which one of the foregrounds was dominant; structures were typically on a scale of $\sim 1^{\circ}$ to $5^{\circ}$. At each WMAP frequency a simultaneous cross-correlation was made of the WMAP emission with the three templates - H-alpha (Dickinson et al., 2003) for free-free, $408 \mathrm{MHz}$ (Haslam et al., 1982) for synchrotron and the Finkbeiner, Davis \& Schlegel (1999) model 8 for dust. Figure 1 shows the WMAP K-band images along with the H-alpha, 100 micron dust and $408 \mathrm{MHz}$ images in 3 fields. Region 6 demonstrates the very close correspondence between the $\mathrm{K}$-band and dust images - there is no evidence for active star formation in the individual clouds shown by the $\mathrm{H}$-alpha or the $408 \mathrm{MHz}$ emission. Although 5 regions were chosen to have dominant dust emission, all 15 regions showed significant dust-correlated emission in the WMAP bands.

Figure 3 shows the temperature spectrum relative to FDS dust model 8 at $94 \mathrm{GHz}$ (Wband). It can be seen that the spectra of the individual features have a similar shape, but there is a spread of a factor of $\sim 2.5$ from feature to feature. This spread can be understood in terms of a dust emissivity which varies with dust temperature as $T_{D}{ }^{1.6}$. When corrected for a thermal spectral index of $\beta=+1.7$, the anomalous dust spectrum has an index of $\beta=-2.85$ between $K$ and $\mathrm{Q}$ bands as shown in Figure 2. The steepening of the spectral index above $33 \mathrm{GHz}(\mathrm{Ka}$ band) is expected on spinning dust models.

It is interesting to compare these results with those of the WMAP team whose MEM solution referred to the whole sky including the Galactic plane modelled as a plane-parallel slab. 
The 15 regions were isolated intermediate Galactic latitude features decomposed into three foregrounds using a cross-correlation technique. When full-sky fits outside the Kp2 cut (i.e. removing the Galactic plane) a similar dust emissivity spectrum was found, but with values $\sim 15 \%$ less than the average of the 15 selected regions. This implies that the selected regions are typical of the intermediate latitude sky but with slightly lower emissivity - perhaps due to cooler dust.

\section{Compact dust clouds}

Compact isolated dust clouds provide the best opportunity to study the physical properties of anomalous emission. Watson et al. (2005) discovered strong emission in the Perseus Molecular Cloud (G159.6-18.5) lying at a distance of $260 \mathrm{pc}$ with the COSMOSOMAS experiment operating in the frequency range 11 to $17 \mathrm{GHz}$. The radio emission was associated with an IRAS ring $\sim 1 .{ }^{\circ} 5$ in diameter. A low surface brightness HII region with a flux density of $\sim 7 \mathrm{Jy}$ underlies the dust emission. Fig. 3 shows the integrated spectrum of the region based on the COSMOSOMAS, WMAP, 0.408 and $1.4 \mathrm{GHz}$ data. A composite Draine \& Lazarian model is shown for comparison, indicating an encouraging fit to a spinning dust model. Recent observations with the Super-Extended VSA at $33 \mathrm{GHz}$ with 7 arcmin resolution (see poster by R.A. Watson et al. at this meeting) show clear emission from features in the IRAS ring. The 33 $\mathrm{GHz}$ emission shows strongest correlation with the $25 \mu$ IRAS band suggesting that the emission is from very small grains.

A morphological study of the dark cloud LDN 1622 has been made by Casassus et al. (2005) using the CBI which has a resolution of 8 arcmin at $31 \mathrm{GHz}$. LDN 1622 lies in the Orion East molecular cloud at $\sim 120 \mathrm{pc}$; it has a star-less core and has structure on a scale of $<10$ arcmin within a 20'x30' region. Finkbeiner et al. (2002) found a rising spectrum from 5 to $10 \mathrm{GHz}$. The integrated free-free emission from the region is $<0.1 \mathrm{Jy}$ which is negligible compared with the dust emission at $31 \mathrm{GHz}$ of $2.9 \mathrm{Jy}$. When combined with the WMAP data, a spectrum peaked at $\sim 30 \mathrm{GHz}$ is seen, similar to that expected for spinning dust. The morphological analysis of $31 \mathrm{GHz}$ and the IRAS data shows a close correlation with all bands but is tightest with the $25 \mu$ band again suggesting the presence of very small grains.

\section{Dust in HII regions}

Significant amounts of dust are found in HII regions. Dust temperatures here are higher than in the general ISM discussed above - in the range 20 to $30 \mathrm{~K}$. Very small grains are not believed to exist in the HII region environment. It is therefore important to clarify the situation with regard to anomalous emission from dust in HII regions.

Dickinson et al. (2006) have observed the diffuse HII region LPH96 (G201.66+1.64) with the $\mathrm{CBI}$ at $31 \mathrm{GHz}$ with a resolution of $\sim 6$ arcmin. A collation of existing data shows that the radio spectrum is mainly free-free emission. 14 percent of the flux density could be 
anomalous emission at $31 \mathrm{GHz}$; the corresponding $31 \mathrm{GHz}$ emissivity relative to $100 \mu$ is somewhat less than for the ISM dust described in Sections 2, 3 and 4.

Casassus et al. (2004) have found $31 \mathrm{GHz}$ emission in excess of the free-free level from the evolved Helix planetary nebula. Since there are not PAH features in the IR spectrum of this nebula, the authors consider that the anomalous emission might arise from magnetic dipole emission from hot ferromagnetic grains.

\section{Polarization of anomalous emission}

Since the anomalous dust gives the dominant foreground to the CMB in the frequency range 10$70 \mathrm{GHz}$ it is critical to know its polarization properties for current and up-coming measurements of CMB polarization. The first attempts have been made. Battistella et al. (2006) have measured the linear polarization of the Perseus Molecular Cloud at a frequency of $11 \mathrm{GHz}$ using COSMOSOMAS; they find $\mathrm{P}=3.4+1.4 /-1.8$ percent at $95 \%$ confidence level. This measurement sets a strong upper limit of 5 percent. The CBI measurements of dust in the diffuse HII region LPH96 set a 2 sigma limit of 10 percent polarization in the anomalous emission. Further work in this area is highly desirable.

\section{Conclusions}

Anomalous dust emission has been seen in individual dust clouds with the properties expected of spinning dust - a peaked emission spectrum and association with very small grain dust. There is also evidence for dust emissivity to increase with temperature.

The anomalous dust in the WMAP bands is widespread at intermediate and high Galactic latitudes. It is the dominant CMB foreground at WMAP frequencies; further work to understand this foreground is critical for future major CMB projects. This work includes low frequency observations to define the anomalous spectrum, definitive polarization measurements and studies of dust in HII regions.

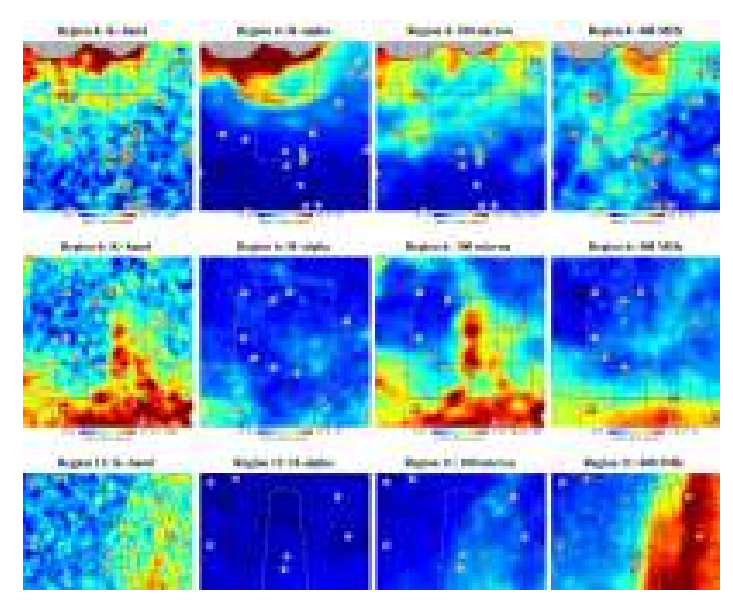

Figure 1 Maps of 3 regions comparing WMAP $\mathrm{K}$ band, $\mathrm{H}-$ alpha, 100 micron and $408 \mathrm{MHz}$. Region 4 has dominant free-free (H-alpha), region 6 has dominant 100 micron dust while region 1 has dominant synchrotron (408 $\mathrm{MHz}$ ). Each region shown is $25^{\circ} \times 25^{\circ}$; the dotted black/white lines show the areas used in the analysis. The small circles mask extragalactic sources. From Davies et al. 2006. 


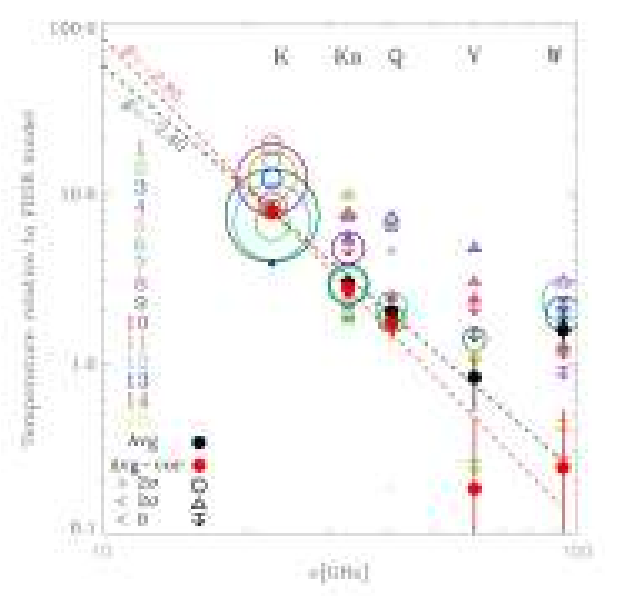

Figure 2 The WMAP dust emissivities of 15 regions relative to FDS $100 \mu$ scaled to 94 GHz. A larger circle indicates higher significance. The weighted average emissivity in each band is plotted by a filled black circle; when corrected for thermal dust emission $(\beta=+1.7)$ it is plotted as a red filled circle. Note that the corrected emissivity has a slope of -2.85 between $\mathrm{K}$ and $\mathrm{Q}$ then steepens above Q. From Davies et al. 2006

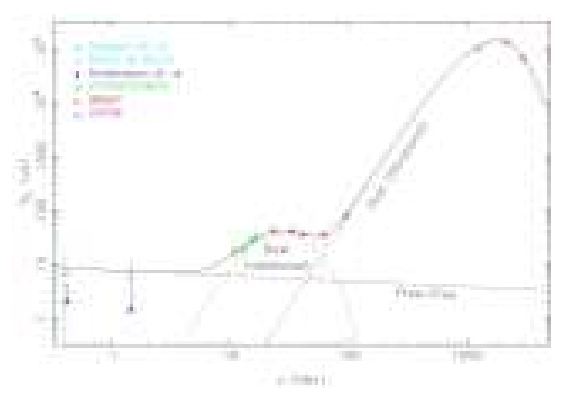

Figure 3 The spectrum G159.618.5 in the Perseus Molecular Cloud. Data are from COSMOSOMAS, WMAP, DIRBE and low frequency surveys. Free-free emission underlies the anomalous emission which peaks at $\sim 30 \mathrm{GHz}$. A Draine \& Lazarian spinning dust model is shown for comparison. From Watson et al. 2005.

\section{References}

[1] Agladze N.I., et al., 1996, ApJ, 462, 1026

[2] Agladze N.I., Sievers A.J., 1998, Phys.Rev.Lett., 80, 4209

[3] Battistelli E.S., et al., 2006, ApJ in press

[4] Banday A.J., Dickinson C., Davies R.D., Davis R.J., Gorski K.M., 2003, MNRAS, 345, 897

[5] Bennett C.L., et al., 2003, ApJS, 148, 97

[6] Boughn S., Cheng E.S., Cottingham D.A., Fixen D.J., 1992 ApJ, 391, L49

[7] Casassus S., Readhead, A.C.S., Pearson T.J., Nyman L-A, Shepherd M.C., Bronfman L., 2004, ApJ, 603, 599

[8] Casassus S., Cabrera G., Förster F., Pearson T.J., Readhead A.C.S., 2006, ApJ 639, 951 
[9] Davies R.D., Dickinson C., Banday A.J., Jaffe T.R., Gorski K.M., Davis R.J., 2006, MNRAS, in press

[10]De Oliveira-Costa A., et al., 1997, ApJ, 482, L17

[11]De Oliveira-Costa A., et al., 1999, ApJ, 527, L9

[12] De Oliveira-Costa A., et al., 2004, ApJ, 606, L89

[13] Dickinson C., Davies R.D., Davis R.J., 2003, MNRAS, 341, 369

[14]Dickinson C., Casassus S., Pineda J.L., Pearson T.J., Readhead A.C.S., Davies R.D., 2006, ApJ in press

[15] Draine B.T., Lazarian A., 1998, ApJ, 494, L19

[16] Draine B.T., Lazarian A., 1999, ApJ, 512, 740

[17] Finkbeiner D.P., Davis M., Schlegel D.J., 1999, ApJ, 524, 867 (FDS)

[18]Finkbeiner D.P., Schlegel D.J., Frank C., Heiles C., 2002, ApJ, 566, 898

[19] Haslam C.G.T., Stoffel H., Salter, C.J., Wilson W.E., 1982, A\&AS, 47, 1

[20] Hinshaw G. et al., 2006, ApJ, in press

[21] Kogut A., et al., 1996a, ApJ, 464, 1

[22] Kogut A., et al., 1996b, ApJ, 464, L5

[23] Lagache G., 2003, A\&A, 405, 813

[24] Leitch E.M., Readhead A.C.S., Pearson T.J., Myers S.T., 1997, ApJ, 486, L23

[25] Leitch E.M., et al., 2000, ApJ, 532, L37

[26] Page L., et al., 2006, ApJ, in press

[27] Watson R.A., et al., 2005, ApJ, 624, L89 